\title{
MANCHESTER
}

1824

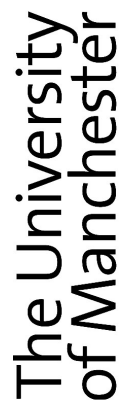

\section{ON THE DIMENSION OF PRODUCTS OF HOMOGENEOUS SUBSPACES IN FREE LIE ALGEBRAS}

\author{
Mansuroglu, Nil and Stöhr, Ralph
}

2013

MIMS EPrint: 2012.49

Manchester Institute for Mathematical Sciences

School of Mathematics

The University of Manchester

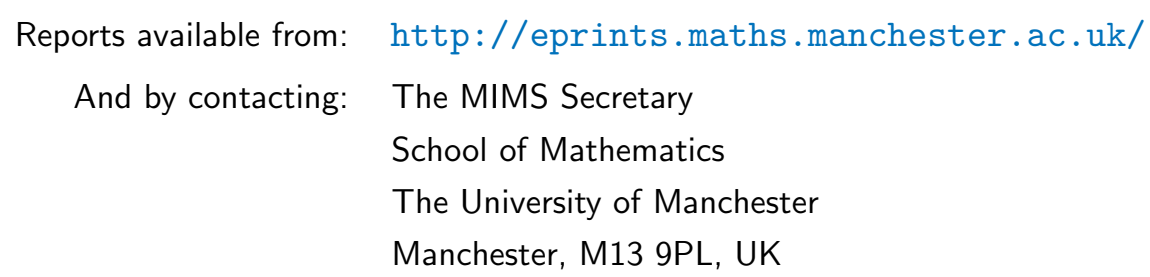

ISSN 1749-9097 


\title{
ON THE DIMENSION OF PRODUCTS OF HOMOGENEOUS SUBSPACES IN FREE LIE ALGEBRAS
}

\author{
NIL MANSUROĞLU* and RALPH STÖHR ${ }^{\dagger}$ \\ School of Mathematics University of Manchester \\ Alan Turing Building, Manchester, M13 9PL, UK \\ *nil.mansuroglu@postgrad.manchester.ac.uk \\ ${ }^{\dagger}$ Ralph.Stohr@manchester.ac.uk
}

Received 10 May 2012

Accepted 13 December 2012

Published 18 February 2013

Communicated by O. Kharlampovich

\begin{abstract}
Let $L$ be a free Lie algebra of finite rank over a field $K$ and let $L_{n}$ denote the degree $n$ homogeneous component of $L$. Formulae for the dimension of the subspaces $\left[L_{m}, L_{n}\right]$ for all $m$ and $n$ were obtained by the second author and Michael Vaughan-Lee. In this note we consider subspaces of the form $\left[L_{m}, L_{n}, L_{k}\right]=\left[\left[L_{m}, L_{n}\right], L_{k}\right]$. Surprisingly, in contrast to the case of a product of two homogeneous components, the dimension of such products may depend on the characteristic of the field $K$. For example, the dimension of $\left[L_{2}, L_{2}, L_{1}\right]$ over fields of characteristic 2 is different from the dimension over fields of characteristic other than 2. Our main results are formulae for the dimension of $\left[L_{m}, L_{n}, L_{k}\right]$. Under certain conditions on $m, n$ and $k$ they lead to explicit formulae that do not depend on the characteristic of $K$, and express the dimension of $\left[L_{m}, L_{n}, L_{k}\right]$ in terms of Witt's dimension function.
\end{abstract}

Keywords: Free Lie algebras; homogeneous subspaces.

Mathematics Subject Classification: 17B01

\section{Introduction}

Let $L$ be a free Lie algebra of rank $r$ over a field $K$. We let $L_{n}$ denote the degree $n$ homogeneous component of $L$, that is the subspace spanned by Lie products of degree $n$ in the free generators of $L$. Then

$$
L=L_{1} \oplus L_{2} \oplus \cdots \oplus L_{n} \oplus \cdots .
$$

The dimension of $L_{n}$ is given by Witt's formula

$$
\operatorname{dim} L_{n}=f(n, r)=\frac{1}{n} \sum_{d \mid n} \mu(d) r^{n / d},
$$


where $\mu$ is the Möbius function (see [8; 4, Theorem 5.11]). In [7] the second author and Vaughan-Lee obtained formulae for the dimensions of the subspaces $\left[L_{m}, L_{n}\right] \leq$ $L_{m+n}$ for all $m, n \geq 1$. They proved that, if $m>n$ and $n \nmid m$, then

$$
\operatorname{dim}\left(\left[L_{m}, L_{n}\right]\right)=\operatorname{dim} L_{m} \operatorname{dim} L_{n},
$$

and if $m=s n$ with $s \geq 1$, then

$$
\operatorname{dim}\left(\left[L_{m}, L_{n}\right]\right)=\left(\operatorname{dim} L_{m}-f\left(s, \operatorname{dim} L_{n}\right)\right) \operatorname{dim} L_{n}+f\left(s+1, \operatorname{dim} L_{n}\right) .
$$

In this paper we investigate the dimension of subspaces of the form $\left[L_{m}, L_{n}, L_{k}\right]$. Throughout the paper we use the left-normed convention for Lie brackets, that is $\left[a_{1}, a_{2}, \ldots, a_{i}\right]=\left[\left[a_{1}, a_{2}, \ldots, a_{i-1}\right], a_{i}\right]$ for $a_{1}, \ldots, a_{i} \in L$, in particular, $\left[L_{m}, L_{n}, L_{k}\right]=\left[\left[L_{m}, L_{n}\right], L_{k}\right]$. It turns out that, somewhat surprisingly and in contrast to products of two homogeneous components, the dimension of $\left[L_{m}, L_{n}, L_{k}\right]$ may depend on the field $K$. In Sec. 4 we use a result of Kuz'min's [3] to show that for $r \geq 5$ the dimension of $\left[L_{2}, L_{2}, L_{1}\right]$ over a field of characteristic 2 is different from the dimension over a field of characteristic other than 2. Moreover, we deduce explicit formulae for the dimension of this space. Before that, in Sec. 3, we derive formulae for the dimension of $\left[L_{m}, L_{n}, L_{k}\right]$ under certain conditions on $m, n$ and $k$. In some cases they lead to explicit formulae that do not depend on the characteristic of $K$ and express the dimension of $\left[L_{m}, L_{n}, L_{k}\right]$ in terms of Witt's dimension function $f(n, r)$. In Sec. 2 we prove our main lemma, a result about the dimension of a product of two arbitrary homogeneous subspaces in $L$. This is a generalization of the main result of [7], and the formulae (1.1) and (1.2) follow easily from it (see Corollary 2.1).

\section{Products of Two Homogeneous Subspaces}

For a subset $Y \subseteq L$ we let $L(Y)$ denote the Lie subalgebra generated by $Y$ in $L$. By the Shirshov-Witt theorem, any subalgebra of $L$ is itself a free Lie algebra $[5,9]$. We write $L_{n}(Y)$ for the degree $n$ homogeneous component of $L(Y)$ (with the degree defined with respect to some free generating set of $L(Y)$ ). The main ingredient of the proof of our main lemma below is a powerful result of Shirshov's. This result, commonly referred to as Shirshov's Lemma, was in fact the key step in Shirshov's original proof of the Shirshov-Witt theorem [5]. An English translation of this celebrated paper is now available in [6]. We record a special case of Shirshov's lemma. A set $Y$ of homogeneous elements in $L$ is called reduced if no element of $Y$ is contained in the subalgebra of $L$ generated by the other elements of $Y$.

Lemma 2.1 ([5, Proof of Theorem 2]). If $L$ is a free Lie algebra and if $Y$ is a reduced set of homogeneous elements in $L$, then $Y$ is a set of free generators for the subalgebra $L(Y)$.

We mention that the elements of $Y$ are required to be homogeneous, but that there is no requirement that they all have the same degree. An immediate consequence of the lemma is that for any homogeneous subspace $U \subseteq L_{m}$, the Lie 
subalgebra $L(U)$ is free of $\operatorname{rank} \operatorname{dim} U$, and any $K$-basis of $U$ is a free generating set for $L(U)$.

Lemma 2.2. Let $U$ and $V$ be subspaces of $L$ such that $U \subseteq L_{m}, V \subseteq L_{n}$ with $m \geq n \geq 1$. Then

$$
\operatorname{dim}[U, V]=\operatorname{dim}[U \cap L(V), V]+(\operatorname{dim} U-\operatorname{dim}(U \cap L(V))) \operatorname{dim} V .
$$

Proof. Let $\mathcal{A}$ be a $K$-basis of $V$. Then $\mathcal{A}$ is a reduced set, and hence a free generating set for the subalgebra $L(V)$. Consider the intersection $U \cap L(V)$, and let $U^{\prime}$ be a complement of $U \cap L(V)$ in $U$, so that $U=(U \cap L(V)) \oplus U^{\prime}$ as a $K$-space. Let $\mathcal{B}$ be a $K$-basis of $U^{\prime}$. We claim that the union $\mathcal{A} \cup \mathcal{B}$ is a reduced set. If $m=n$ then $\mathcal{A} \cup \mathcal{B}$ is a linearly independent subset of $L_{n}$, and hence reduced. Now assume that $m>n$. Then the elements in $\mathcal{B}$ have larger degree than the elements in $\mathcal{A}$, so the only way an element $a \in \mathcal{A}$ could be in the subalgebra generated by the other elements of $\mathcal{A} \cup \mathcal{B}$ would be if $a$ was a linear combination of the other elements of $\mathcal{A}$. But this is impossible since $\mathcal{A}$ is linearly independent. On the other hand, the only way an element $b \in \mathcal{B}$ could be in the subalgebra generated by the other elements of $\mathcal{A} \cup \mathcal{B}$ would be if

$$
b=v+\sum_{b_{i} \in \mathcal{B}, b_{i} \neq b} \alpha_{i} b_{i}
$$

for some $v \in L(V)$ and some scalars $\alpha_{i}$. But this is impossible by the choice of $\mathcal{B}$. Indeed, (2.2) implies that $v$ is a linear combination of the elements in $\mathcal{B}$. Hence $v=0$. But then $b$ is a linear combination of the other elements in $\mathcal{B}$ which is not possible as $\mathcal{B}$ is linearly independent. Hence $\mathcal{A} \cup \mathcal{B}$ is a reduced set. It follows that the set $\{[b, a] ; b \in \mathcal{B}, a \in \mathcal{A}\}$ is linearly independent. Hence it is a basis for the product $\left[U^{\prime}, V\right]$, and

$$
\operatorname{dim}\left[U^{\prime}, V\right]=(\operatorname{dim} U-\operatorname{dim}(U \cap L(V))) \operatorname{dim} V .
$$

Moreover, we can write $[U, V]$ as

$$
[U, V]=\left[(U \cap L(V)) \oplus U^{\prime}, V\right]=[(U \cap L(V)), V]+\left[U^{\prime}, V\right] .
$$

But the sum on the right-hand side in (2.3) is direct since the first summand is in $L(V)=L(\mathcal{A})$, and the second is in the ideal generated by $\mathcal{B}$ in the free Lie algebra $L(\mathcal{A} \cup \mathcal{B})$. So

$$
[U, V]=[(U \cap L(V)), V] \oplus\left[U^{\prime}, V\right]
$$

and this yields (2.1), as required.

The main result of [7] is an immediate consequence of this lemma.

Corollary 2.1 ([7]). Let $m \geq n \geq 1$. If $n \nmid m$, then the dimension of the subspace $\left[L_{m}, L_{n}\right]$ is given by (1.1), and if $m=$ sn with $s \geq 1$, then the dimension of this subspace is given by (1.2). 
Proof. Apply Lemma 2.2 with $U=L_{m}$ and $V=L_{n}$. If $n \nmid m$, then $L\left(L_{n}\right) \cap L_{m}=0$, whence (1.1). If $m=s n$ with $s \geq 1$, then $L\left(L_{n}\right) \cap L_{m}=L_{s}\left(L_{n}\right)$ and, of course, $\left[L_{s}\left(L_{n}\right), L_{n}\right]=L_{s+1}\left(L_{n}\right)$, whence $(1.2)$.

\section{Products of Three Homogeneous Components}

We begin this section with a technical lemma.

Lemma 3.1. Let $m, n, k$ be positive integers.

(i) If $m=s k$ and $n=t k$ for some positive integers $s$ and $t$, then

$$
\left[L_{m}, L_{n}\right] \cap L\left(L_{k}\right)=\left[L_{s}\left(L_{k}\right), L_{t}\left(L_{k}\right)\right] .
$$

(ii) If $k \nmid n$ or $k \nmid m$, then

$$
\left[L_{m}, L_{n}\right] \cap L\left(L_{k}\right)=0 .
$$

Proof. (i) Obviously, $\left[L_{s}\left(L_{k}\right), L_{t}\left(L_{k}\right)\right] \subseteq\left[L_{m}, L_{n}\right] \cap L\left(L_{k}\right)$. We need to prove the inverse inclusion.

Consider the subalgebra

$$
L^{k}=L_{k} \oplus L_{k+1} \oplus L_{k+2} \oplus \cdots,
$$

that is the $k$ th term of the lower central series of $L$. It is well known that $L^{k}$ has a homogeneous free generating set of the form $\mathcal{C}=\mathcal{C}_{k} \cup \mathcal{C}_{k+1} \cup \mathcal{C}_{k+2} \cup \cdots$ where $\mathcal{C}_{i} \subset L_{i}(i=k, k+1, \ldots)$. In fact, such a free generating set can easily be obtained as follows. We take a $K$-basis of $L_{k}$ for $\mathcal{C}_{k}$, and, for $i>k$, we proceed by induction taking a basis of a vector space complement of $L_{i} \cap L\left(\mathcal{C}_{k} \cup \cdots \cup \mathcal{C}_{i-1}\right)$ as the set $\mathcal{C}_{i}$. It is obvious that the resulting set is a generating set for $L^{k}$, and Shirshov's lemma guarantees that it is a free generating set. This free generating set is such that $L_{k}=\left\langle\mathcal{C}_{k}\right\rangle$ and $L\left(L_{k}\right)$ is freely generated by $\mathcal{C}_{k}$. Let $\pi: L^{k} \rightarrow L\left(L_{k}\right)$ be the projection map given by $c \pi=c$ for $c \in \mathcal{C}_{k}$ and $c \pi=0$ for $c \in \mathcal{C} \backslash \mathcal{C}_{k}$. Any element in $\left[L_{m}, L_{n}\right]$ is a linear combination of the form $w=\sum \alpha_{j}\left[u_{j}, v_{j}\right]$ with $u_{j} \in L_{m}$, $v_{j} \in L_{n}$ and $\alpha_{j} \in K$. Note that $u_{j} \pi \in L_{s}\left(L_{k}\right)$ and $v_{j} \pi \in L_{t}\left(L_{k}\right)$. Assume that $w \in L\left(L_{k}\right)$. Then, since $\pi$ is the identity on $L\left(L_{k}\right)$ and since $\pi$ is a Lie algebra homomorphism, we have

$$
w=w \pi=\sum_{j} \alpha_{j}\left[u_{j} \pi, v_{j} \pi\right] \in\left[L_{s}\left(L_{k}\right), L_{t}\left(L_{k}\right)\right] .
$$

This proves the required inclusion.

(ii) Now suppose that $k \nmid n$ or $k \nmid m$, and let $w=\sum \alpha_{j}\left[u_{j}, v_{j}\right] \in L\left(L_{k}\right)$ with $u_{j} \in L_{m}$ and $v_{j} \in L_{n}$. We use the projection $\pi$ as introduced above. Since $L\left(L_{k}\right)$ consists entirely of linear combinations of elements of degree $q k$ with $q=1,2,3, \ldots$, all homogeneous components $L_{i}$ with $i \geq k$ and $k \nmid i$ are in the kernel of $\pi$. Hence $\left[u_{j}, v_{j}\right] \pi=\left[u_{j} \pi, v_{j} \pi\right]=0$ for all $j$ since by our assumption at least one of $u_{j} \pi$ and $v_{j} \pi$ is zero. Therefore $\left[L_{m}, L_{n}\right] \cap L\left(L_{k}\right)=0$. 
Now we are ready for our main result.

Theorem 3.1. Let $m, n$ and $k$ be positive integers with $m \geq n$.

(i) If $m+n>k$ and $k \nmid m$ or $k \nmid n$, then

$$
\operatorname{dim}\left[L_{m}, L_{n}, L_{k}\right]=\operatorname{dim}\left[L_{m}, L_{n}\right] \operatorname{dim} L_{k},
$$

(ii) if $m+n>k$ and $m=s k$ and $n=t k$ with $s, t \geq 1$, then

$$
\begin{aligned}
\operatorname{dim}\left[L_{m}, L_{n}, L_{k}\right]= & \operatorname{dim}\left[L_{s}\left(L_{k}\right), L_{t}\left(L_{k}\right), L_{k}\right] \\
& +\left(\operatorname{dim}\left[L_{m}, L_{n}\right]-\operatorname{dim}\left[L_{s}\left(L_{k}\right), L_{t}\left(L_{k}\right)\right]\right) \operatorname{dim} L_{k},
\end{aligned}
$$

(iii) and if $k \geq m+n$ and $(m+n) \nmid k$, then

$$
\operatorname{dim}\left[L_{m}, L_{n}, L_{k}\right]=\operatorname{dim}\left[L_{m}, L_{n}\right] \operatorname{dim} L_{k},
$$

(iv) if $k \geq m+n$ and $k=p(m+n)$ with $p \geq 1$, then

$$
\begin{aligned}
\operatorname{dim}\left[L_{m}, L_{n}, L_{k}\right]= & \operatorname{dim} L_{p+1}\left(\left[L_{n}, L_{m}\right]\right) \\
& +\left(\operatorname{dim} L_{k}-\operatorname{dim}\left(L_{p}\left(\left[L_{m}, L_{n}\right]\right)\right)\right) \operatorname{dim}\left[L_{m}, L_{n}\right] .
\end{aligned}
$$

Proof. (i) We apply Lemma 2.2 with $U=\left[L_{m}, L_{n}\right]$ and $V=L_{k}$. This gives

$$
\begin{aligned}
\operatorname{dim}\left[L_{m}, L_{n}, L_{k}\right]= & \operatorname{dim}\left[\left(\left[L_{m}, L_{n}\right] \cap L\left(L_{k}\right)\right), L_{k}\right] \\
& +\left(\operatorname{dim}\left[L_{m}, L_{n}\right]-\operatorname{dim}\left(\left[L_{m}, L_{n}\right] \cap L\left(L_{k}\right)\right)\right) \operatorname{dim} L_{k},
\end{aligned}
$$

and the result follows since $\left[L_{m}, L_{n}\right] \cap L\left(L_{k}\right)=0$ by Lemma 3.1(ii).

(ii) Again, we apply Lemma 2.2 with $U=\left[L_{m}, L_{n}\right]=\left[L_{s k}, L_{t k}\right]$ and $V=L_{k}$. This gives

$$
\begin{aligned}
\operatorname{dim}\left[L_{m}, L_{n}, L_{k}\right]= & \operatorname{dim}\left[\left(\left[L_{s k}, L_{t k}\right] \cap L\left(L_{k}\right)\right), L_{k}\right] \\
& +\left(\operatorname{dim}\left[L_{m}, L_{n}\right]-\left(\operatorname{dim}\left(\left[L_{s k}, L_{t k}\right] \cap L\left(L_{k}\right)\right)\right)\right) \operatorname{dim} L_{k} .
\end{aligned}
$$

By Lemma 3.1(i), $\left[L_{s k}, L_{t k}\right] \cap L\left(L_{k}\right)=\left[L_{s}\left(L_{k}\right), L_{t}\left(L_{k}\right)\right]$. Hence

$$
\begin{aligned}
\operatorname{dim}\left[L_{m}, L_{n}, L_{k}\right]= & \operatorname{dim}\left[\left[L_{s}\left(L_{k}\right), L_{t}\left(L_{k}\right)\right], L_{k}\right] \\
& +\left(\operatorname{dim}\left[L_{m}, L_{n}\right]-\operatorname{dim}\left[L_{s}\left(L_{k}\right), L_{t}\left(L_{k}\right)\right]\right) \operatorname{dim} L_{k},
\end{aligned}
$$

as required.

(iii) Of course, $\left[L_{m}, L_{n}, L_{k}\right]=\left[L_{k},\left[L_{m}, L_{n}\right]\right]$. We apply Lemma 2.2 with $U=L_{k}$ and $V=\left[L_{m}, L_{n}\right]$. This gives

$$
\begin{aligned}
\operatorname{dim}\left[L_{m}, L_{n}, L_{k}\right]= & \operatorname{dim}\left[\left(L_{k} \cap L\left(\left[L_{m}, L_{n}\right]\right)\right),\left[L_{m}, L_{n}\right]\right] \\
& +\left(\operatorname{dim} L_{k}-\operatorname{dim}\left(L_{k} \cap L\left(\left[L_{m}, L_{n}\right]\right)\right)\right) \operatorname{dim}\left[L_{m}, L_{n}\right] .
\end{aligned}
$$

Since $m+n \nmid k, L_{k} \cap L\left(\left[L_{m}, L_{n}\right]\right)=0$, and (3.1) turns into the formula in part (iii) of the theorem. 
(iv) As in (iii), we apply Lemma 2.2 with $U=L_{k}$ and $V=\left[L_{m}, L_{n}\right]$. Here we have $L_{k} \cap L\left(\left[L_{m}, L_{n}\right]\right)=L_{p(m+n)} \cap L\left(\left[L_{m}, L_{n}\right]\right)=L_{p}\left(\left[L_{m}, L_{n}\right]\right)$, so (3.1) turns into

$$
\begin{aligned}
\operatorname{dim}\left[L_{m}, L_{n}, L_{k}\right]= & \operatorname{dim}\left[L_{p}\left(\left[L_{m}, L_{n}\right]\right),\left[L_{m}, L_{n}\right]\right] \\
& +\left(\operatorname{dim} L_{k}-\operatorname{dim} L_{p}\left(\left[L_{m}, L_{n}\right]\right)\right) \operatorname{dim}\left[L_{m}, L_{n}\right],
\end{aligned}
$$

and the result follows since $\left[L_{p}\left(\left[L_{m}, L_{n}\right]\right),\left[L_{m}, L_{n}\right]\right]=L_{p+1}\left(\left[L_{m}, L_{n}\right]\right)$.

\section{An Example}

Our main result, Theorem 3.1, gives formulae for the dimension of subspaces of the form $\left[L_{m}, L_{n}, L_{k}\right]$ under various conditions on $m, n$ and $k$. Since we have explicit formulae for the dimension of $\left[L_{m}, L_{n}\right]$ for all $m, n$, namely formulae (1.1) and (1.2), all but one of the terms on the right-hand sides of the four formulae in Theorem 3.1 can be expressed in terms of Witt's dimension function $f(n, r)$. The exception is $\operatorname{dim}\left[L_{s}\left(L_{k}\right), L_{t}\left(L_{k}\right), L_{k}\right]$ in the formula in part (ii) of the theorem. It appears to be considerably harder to work out the dimension under the conditions in part (ii). The obstacles here are the products $\left[L_{s}\left(L_{k}\right), L_{t}\left(L_{k}\right), L_{k}\right]$. The smallest possible instance of such a product is $\left[L_{2}, L_{2}, L_{1}\right]$. It turns out that its dimension depends on the characteristic of the ground field.

Proposition 4.1. Let $L$ be the free Lie algebra of rank $r$ over a field $K$. If $r \geq 5$, then the dimension of $\left[L_{2}, L_{2}, L_{1}\right]$ over a field of characteristic 2 is strictly less than the dimension of $\left[L_{2}, L_{2}, L_{1}\right]$ over a field of characteristic other than 2 .

Proof. This is an immediate consequence of a result by Kuz'min [3] on the free center-by-metabelian Lie ring. Let $\mathfrak{L}$ denote the free Lie ring on free generators $x_{1}, x_{2}, \ldots, x_{r}, r \geq 2$, and write $\mathfrak{L}_{n}$ for its degree $n$ homogeneous component. The free center-by-metabelian Lie ring is the quotient $\mathfrak{G}=\mathfrak{L} /\left[\mathfrak{L}^{\prime \prime}, \mathfrak{L}\right]$, where $\mathfrak{L}^{\prime \prime}$ is the second term of the derives series of $\mathfrak{L}$. Then $\mathfrak{G}$ is a graded ring, and we denote its degree $n$ homogeneous component by $\mathfrak{G}_{n}$. Here $\mathfrak{G}_{n} \cong \mathfrak{L}_{n} /\left(\mathfrak{L}_{n} \cap\left[\mathfrak{L}^{\prime \prime}, \mathfrak{L}\right]\right)$. We are interested in $\mathfrak{G}_{5} \cap \mathfrak{G}^{\prime \prime}$. It is easily seen that $\mathfrak{L}_{5} \cap \mathfrak{L}^{\prime \prime}=\left[\mathfrak{L}_{3}, \mathfrak{L}_{2}\right]$ and $\mathfrak{L}_{5} \cap\left[\mathfrak{L}^{\prime \prime}, \mathfrak{L}\right]=$ $\left[\mathfrak{L}_{2}, \mathfrak{L}_{2}, \mathfrak{L}_{1}\right]$. Hence

$$
\mathfrak{G}_{5} \cap \mathfrak{G}^{\prime \prime} \cong\left[\mathfrak{L}_{3}, \mathfrak{L}_{2}\right] /\left[\mathfrak{L}_{2}, \mathfrak{L}_{2}, \mathfrak{L}_{1}\right]
$$

By [3, Theorem 4],

$$
\mathfrak{G}_{5} \cap \mathfrak{G}^{\prime \prime}=A \oplus T
$$

where $A$ is a free abelian group which is freely generated by the canonical images in $\mathfrak{G}$ of the Lie polynomials

$$
\left[\left[x_{i}, x_{j}\right],\left[x_{k}, x_{l}, x_{m}\right]\right]
$$

with $i, j, k, l, m \in\{1,2, \ldots, r\}$ and $i>j, k>l, i \geq k, j \geq l$ and $m \geq j$, and $T$ is an elementary abelian 2-group of rank $\left(\begin{array}{l}r \\ 5\end{array}\right)$ if $r \geq 5$ and $T=0$ if $r<5$. Alternatively, 
this also follows from [1, Theorem 1], which relies on [2, Lemma 4.1] for the crucial fact that $\mathfrak{G}_{5} \cap \mathfrak{G}^{\prime \prime}$ contains non-trivial 2-torsion for $r \geq 5$. Tensoring with a field $K$ gives the corresponding result for free center-by-metabelian Lie algebras. In view of (4.1) and (4.2) we find that for the free Lie algebra $L$ of rank $r$ over a field $K$ one has

$$
\operatorname{dim}\left[L_{2}, L_{2}, L_{1}\right]= \begin{cases}\operatorname{dim}\left[L_{3}, L_{2}\right]-\operatorname{rank} A & \text { if } \operatorname{char} K \neq 2, \\ \operatorname{dim}\left[L_{3}, L_{2}\right]-\operatorname{rank} A-\operatorname{rank} T & \text { if } \operatorname{char} K=2\end{cases}
$$

The result follows.

In fact, it is not too difficult to work out the dimension of $\left[L_{2}, L_{2}, L_{1}\right]$ explicitly. First we count the number of distinct Lie products (4.3). We call these Lie polynomials Kuz'min commutators.

Lemma 4.1. The number of distinct Kuz'min commutators is $r\left(\begin{array}{c}r+2 \\ 4\end{array}\right)$.

Proof. We use induction on $r$. The lemma is true for $r=2$ as the only Kuz'min commutators in this case are $\left[\left[x_{2}, x_{1}\right],\left[x_{2}, x_{1}, x_{1}\right]\right]$ and $\left[\left[x_{2}, x_{1}\right],\left[x_{2}, x_{1}, x_{2}\right]\right]$. Now let $r>2$. By induction, the number of Kuz'min commutators that do not involve $x_{1}$ is $(r-1)\left(\begin{array}{c}r+1 \\ 4\end{array}\right)$. To that we need to add the number of Kuz'min commutators that do involve $x_{1}$. If $x_{1}$ is present, it must occur as the fourth entry. Hence these polynomials are of the form

$$
\left[\left[x_{i}, x_{j}\right],\left[x_{k}, x_{1}, x_{m}\right]\right]
$$

with

$$
i \geq k>1, \quad j \leq m
$$

and

$$
i>j .
$$

First we count the commutators (4.5) satisfying conditions (4.6). In these polynomials $(i, k)$ can be any pair of subscripts from $\{2,3, \ldots, r\}$ with $i \geq k$. The number of such pairs is $(r-1) r / 2$. The entries in the second pair of subscripts $(j, m)$ can be any numbers with $1 \leq j \leq m \leq r$. The number of such pairs is $r(r+1) / 2$. Hence the number of commutators (4.5) satisfying (4.6) is

$$
\frac{1}{4}(r-1) r^{2}(r+1)
$$

In order to find the number of Kuz'min commutators involving $x_{1}$ we need to subtract from (4.8) the number of commutators (4.3) satisfying the conditions (4.6) but not (4.7), that is

$$
1<x_{k} \leq x_{i} \leq x_{j} \leq x_{m}
$$


The number of such elements is equal to $\left(\begin{array}{c}r+2 \\ 4\end{array}\right)$. Consequently, the number of Kuz'min commutators involving $x_{1}$ is

$$
\frac{1}{4}\left(r^{2}-1\right) r^{2}-\left(\begin{array}{c}
r+2 \\
4
\end{array}\right)
$$

Now we get the total number of Kuz'min commutators by adding this number to $(r-1)\left(\begin{array}{c}r+1 \\ 4\end{array}\right)$, which is the number of Kuz'min commutators not involving $x_{1}$ :

$$
(r-1)\left(\begin{array}{c}
r+1 \\
4
\end{array}\right)+\frac{1}{4}(r-1) r^{2}(r+1)-\left(\begin{array}{c}
r+2 \\
4
\end{array}\right) .
$$

An elementary calculation shows that this is equal to $r\left(\begin{array}{c}r+2 \\ 4\end{array}\right)$, as required.

Now,

$$
\operatorname{dim}\left[L_{3}, L_{2}\right]-\operatorname{rank} A=\frac{1}{6}\left(r^{3}-r\right)\left(r^{2}-r\right)-r\left(\begin{array}{c}
r+2 \\
4
\end{array}\right)
$$

and

$$
\operatorname{dim}\left[L_{2}, L_{2}\right] \operatorname{dim} L_{1}=\frac{1}{2}\left(\frac{r^{2}-r}{2}\right)\left(\frac{r^{2}-r}{2}-1\right) r .
$$

Another elementary calculation shows that these numbers are equal:

$$
\operatorname{dim}\left[L_{3}, L_{2}\right]-\operatorname{rank} A=\operatorname{dim}\left[L_{2}, L_{2}\right] \operatorname{dim} L_{1} .
$$

It remains to substitute this into (4.4) to get our final result.

Proposition 4.2. Let $L$ be a free Lie algebra of rank $r$ over a field $K$. Then

$$
\operatorname{dim}\left[L_{2}, L_{2}, L_{1}\right]= \begin{cases}\operatorname{dim}\left[L_{2}, L_{2}\right] \operatorname{dim} L_{1} & \text { if } \operatorname{char} K \neq 2, \\
\operatorname{dim}\left[L_{2}, L_{2}\right] \operatorname{dim} L_{1}-\left(\begin{array}{c}
r \\
5
\end{array}\right) & \text { if } \operatorname{char} K=2,\end{cases}
$$

with the convention that $\left(\begin{array}{l}r \\ 5\end{array}\right)=0$ for $r<5$.

\section{References}

[1] N. D. Gupta, T. C. Hurley and F. Levin, On the lower central factors of free centreby-metabelian groups, J. Aust. Math. Soc., Ser. A 38 (1985) 65-75.

[2] N. Gupta and F. Levin, Separating laws for free centre-by-metabelian nilpotent groups, Commun. Algebra 4 (1976) 249-270.

[3] Yu. V. Kuz'min, Free center-by-metabelian groups, Lie algebras and $\mathcal{D}$-groups, Izv. Akad. Nauk SSSR Ser. Mat. 41(1) (1977) 3-33, 231 (in Russian), Math. USSR Izvestija 11(1) (1977) 1-30.

[4] W. Magnus, A. Karrass and D. Solitar, Combinatorial Group Theory. Presentations of Groups in Terms of Generators and Relations, 2nd rev. edn (Dover Publications, New York, 1976).

[5] A. I. Shirshov, Subalgebras of free Lie algebras, Mat. Sb. 33 (1953) 441-452 (in Russian). 
[6] A. I. Shirshov, Selected Works of A. I. Shirshov, Translated from the Russian by Murray Bremner and Mikhail V. Kotchetov, eds. L. A. Bokut, V. Latyshev, I. Shestakov and E. Zelmanov, Contemporary Mathematics (Birkhäuser, Basel, 2009), viii+242 pp.

[7] R. Stöhr and M. Vaughan-Lee, Products of homogeneous subspaces in free Lie algebras, Internat. J. Algebra Comput. 19(5) (2009) 699-703.

[8] E. Witt, Treue Darstellungen Liescher Ringe, J. Reine Angew. Math. 177 (1937) 152160.

[9] E. Witt, Die Unterringe der freien Lieschen Ringe, Math. Z. 64 (1956) 195-216. 